\title{
Burnout among Primary Care Providers and Staff: Evaluating the Association with Practice Adaptive Reserve and Individual Behaviors
}

\author{
Debora Goetz Goldberg, PhD, MHA, MBA ${ }^{\top} \mathbb{D}$, Tulay G. Soylu, PhD, MHA, MBA ${ }^{2}$, \\ Panagiota Kitsantas, $P h D^{7}$, Victoria M. Grady, $P h D^{3}$, Kurt Elward, $M D^{4}$, and \\ Len M. Nichols, Ph.D. ${ }^{5}$
}

\begin{abstract}
'Department of Health Administration and Policy, George Mason University, Fairfax, VA, USA; ${ }^{2}$ Department of Health Services Administration and Policy, Temple University, Philadelphia, PA, USA; ${ }^{3}$ School of Business, George Mason University, Fairfax, VA, USA; ${ }^{4}$ Department of Family Medicine and Population Health, Virginia Commonwealth University, Richmond, VA, USA; ${ }^{5}$ Center for Health Policy Research and Ethics, George Mason University, Fairfax, VA, USA.
\end{abstract}

BACKGROUND: Workplace burnout among healthcare professionals is a critical public health concern. Few studies have examined organizational and individual factors associated with burnout across healthcare professional groups.

OBJECTIVE: The purpose of this study was to examine the association between practice adaptive reserve (PAR) and individual behavioural response to change and burnout among healthcare professionals in primary care.

DESIGN: This cross-sectional study used survey data from 154 primary care practices participating in the EvidenceNOW Heart of Virginia Healthcare initiative.

PARTICIPANTS: We analysed data from 1279 healthcare professionals in Virginia. Our sample included physicians, advanced practice clinicians, clinical support staff and administrative staff.

MAIN MEASURES: We used the PAR instrument to measure organizational capacity for change and the Change Diagnostic Index@ (CDI) to measure individual behavioural response, which achieved a 76\% response rate. Logistic regression analysis was used to estimate the effects of PAR and CDI on burnout.

KEY RESULTS: As organizational capacity for change increased, burnout in healthcare professionals decreased by 51\% (OR: 0.49; 95\% CI, 0.33, 0.73). As healthcare professionals showed improved response toward change, burnout decreased by $84 \%$ (OR: $0.16 ; 95 \%$ CI, $0.11,0.23$ ). Analysis by healthcare professional type revealed a significant association between high organizational capacity for change, positive response to change and low burnout among administrative staff (OR: 2.92; 95\% CI, 1.37, 6.24). Increased hours of work per week was associated with higher odds of burnout (OR: 1.07; 95\% CI, 1.05, 1.10) across healthcare professional groups.

CONCLUSION: As transformation efforts in primary care continue, it is critical to understand the influence of these initiatives on healthcare professionals' well-being. Efforts to reduce burnout among healthcare professionals are

Supplementary Information The online version contains supplementary material available at https://doi.org/10.1007/s11606-020-06367-z.

Received April 29, 2020

Accepted November 25, 2020

Published online January 8, 2021 needed at both a system and organizational level. Building organizational capacity for change, supporting providers and staff during major change and consideration of individual workload may reduce levels of burnout.

KEY WORDS: adaptive reserve; burnout; practice transformation; primary care; well-being.

J Gen Intern Med 36(5):1222-8

DOI: $10.1007 / \mathrm{s} 11606-020-06367-\mathrm{Z}$

(c) Society of General Internal Medicine 2021

\section{INTRODUCTION}

Dramatic changes in the U.S. healthcare system over the past several decades have affected healthcare professionals' roles, responsibilities and work experiences. ${ }^{1}$ Changes include adoption of electronic health records, new care delivery and payment models, increased attention to quality measurement and evidence-based practices and transition of many medical practices from physician owned to ownership by an integrated delivery system. ${ }^{2-5}$ These major transformations intensify job demands and disrupt the work experience, which may negatively influence work-life balance, job control and alignment of professional and personal values. These shifts, and the emotional challenges of change and change fatigue, may contribute to workplace stress and dissatisfaction. ${ }^{6}$

Healthcare professionals who experience chronic workplace stress and those less satisfied with their work environment are more likely to experience burnout. ${ }^{7-9}$ Burnout is a syndrome of emotional exhaustion, depersonalization and reduced personal accomplishment, ${ }^{10}$ which occurs from chronic workplace stress that has not been successfully managed. High levels of burnout among healthcare professionals can lead to physical and mental health problems, high turnover and quality of care issues. ${ }^{1-14}$ Healthcare professionals at highest risk are those at the frontline of healthcare delivery with substantial direct patient care responsibilities, ${ }^{1,15}$ including those that work in primary care physician practices. ${ }^{16}$ 
Workplace burnout among all healthcare professionals is a critical public health concern. ${ }^{17}$ Previous research on burnout has mostly focused on physicians and hospital-based nurses; few studies have evaluated factors associated with burnout across various types of healthcare professionals in primary care settings. ${ }^{1}$ The purpose of this study was to examine the association between practice adaptive reserve (PAR) and individual behavioural response to change and burnout among various types of healthcare professionals in primary care.

\section{METHODS}

The Heart of Virginia Healthcare (HVH) collaborative was part of the Agency for Healthcare Research and Quality's (AHRQ) EvidenceNOW initiative between 2015 and 2018. ${ }^{18}$ The goal of HVH was to support small and medium-sized primary care practices in Virginia with practice transformation and implementation of evidence-based cardiovascular care. Participating in HVH required major changes in practice operations and roles and responsibilities of providers and staff. This study is a cross-sectional analysis of survey data collected during the HVH initiative. The conceptual framework for the study, Figure 1, is adapted from the professional well-being framework from the National Academy of Medicine ${ }^{1}$ and focuses on the relationships between work system factors, individual factors and burnout. The George Mason University Institutional Review Board approved this research in 2016 and methodological updates in 2017.

\section{Data Source}

A member survey was used to measure the practices' adaptive reserve, individual behavioural responses to change, number of hours worked per week and burnout. This survey was administered online and over mail in 2018, which resulted in a response rate of $76 \%$ and included 1279 physicians and staff at 154 practices, out of 1677 individuals contacted. Each individual received $\$ 150$ compensation upon completion of the survey. A separate practice survey was completed by the lead physician or practice manager between 2016 and 2017 that captured information on organizational characteristics. ${ }^{19}$ Participating practices received \$500 compensation for completing the practice survey. Missing data ranged from 0.3 to $0.9 \%$.

\section{Sample}

The sample consisted of 1279 individuals across 154 primary care practices in Virginia that completed member surveys and a practice level survey during the $\mathrm{HVH}$ initiative. The sample included 139 (11.0\%) physicians; 99 (7.8\%) advanced practice clinicians including nurse practitioners and physician assistants; 451 (35.6\%) clinical support staff such as registered nurses, licensed practical nurses, and medical assistants and $578(45.6 \%)$ administrative staff consisting of office managers, receptionists, and billing staff, see Table 1. For data analysis, we grouped the physicians and advanced practice clinicians into a group called "providers" $(n=238)$.

\section{Measures}

The measures used in this study include burnout, work system factors involving adaptive reserve and individual factors concerning healthcare professionals' behavioural response to change; see Appendix A.

Burnout. Burnout was measured by a single item validated instrument used for the Healthy Workplace Trial ${ }^{20}$ and the Physician Worklife Study, ${ }^{21}$ which has been closely aligned to the emotional exhaustion construct of the Maslach Burnout Inventory. ${ }^{22}$ The scale consisted of five possible answers including (1) "I enjoy my work. I have no symptoms of burnout"; (2) “Occasionally I am under stress, and I don't always have as much energy as I once did, but I don't feel burned out"; (3) "I am definitely burning out and have one or more symptoms of burnout, such as physical and emotional exhaustion"; (4) "The symptoms of burnout that I'm experiencing won't go away. I think about frustrations at work a lot" and (5) "I feel completely burned out and often wonder if I can go on practicing. I am at the point where I may need some changes." Responses to 3 or higher on the metric were classified as "burned out."19

Practice Adaptive Reserve. Practice adaptive reserve (PAR) is a framework designed to understand primary care physician practices' capacity for successful change management. ${ }^{23} \mathrm{~A}$ practice's adaptive reserve consists of an institutional core, which includes material and human resources; an organizational structure that describes management and leadership and functional processes, such as clinical care and quality improvement activities. Adaptive reserve also includes organizational attributes supportive of change, such as leadership facilitation, a culture of innovation and learning, and effective relationships and communication. ${ }^{23}$ A strong adaptive reserve is characterized by a positive working environment and teamwork, which has been associated with lower levels of burnout among physicians and other healthcare professionals. ${ }^{24,25} \mathrm{~A}$ strong team culture and team-based care have also been found to be associated with less emotional exhaustion, ${ }^{26}$ an indicator of less burnout.

We used the PAR questionnaire in this study to measure practice members' perceptions of relationship infrastructure, leadership styles and organizational culture. The scale consists of 18 questions on communication, teamwork, relationship trust, practice leadership, work environment and adoption of innovations and learning systems. The PAR instrument is based on a 5-point Likert-type scale (levels ranged from strongly disagree to strongly agree). Example questions include "People in our practice actively seek new ways to improve how we do things," "Practice leadership promotes an environment that is an enjoyable place to work" and "I have 


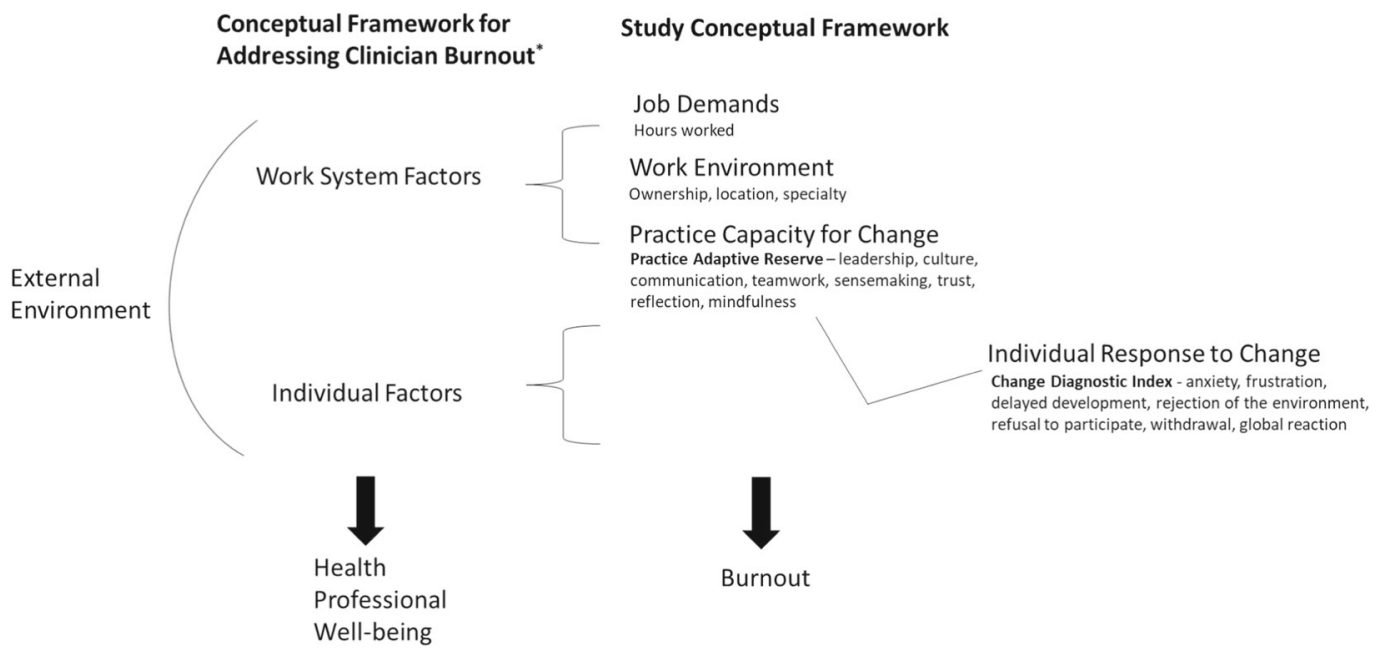

Fig. 1 Study conceptual framework. Adapted from the National Academy of Medicine. Taking action against clinician burnout, A systems approach to professional well-being. Washington, DC; 2019.

many opportunities to grow in my work.” Reliability analysis for the PAR revealed a Cronbach's alpha of 0.93 . The average summed responses of these 18 items were used in the analysis. Higher average scores for the PAR indicate higher capacity for change in practices. Adaptive reserve was measured for each practice at the member level. ${ }^{23}$

Change Diagnostic Index. Individual response to major changes and work-related stressors is an important

Table 1 Sample Characteristics

\begin{tabular}{ll}
\hline \hline & $\boldsymbol{n}=\mathbf{1 2 7 9}^{\S}, \boldsymbol{n} \mathbf{( \% )}$ or mean (SD) \\
\hline Role at the practice & \\
Providers & $238(18.8)$ \\
Clinical support staff ${ }^{\dagger}$ & $451(35.6)$ \\
Administrative staff ${ }^{\dagger}$ & $578(45.6)$ \\
Specific role at the practice & $139(11.0)$ \\
Physicians & $99(7.8)$ \\
Advanced practice clinicians* & $451(35.6)$ \\
Clinical support staff ${ }^{\dagger}$ & \\
Administrative staff ${ }^{\dagger}$ & $358(28.3)$ \\
$\quad$ Receptionists and billing staff & $96(7.6)$ \\
Office managers & $124(9.8)$ \\
Other & $249(19.5)$ \\
Burnout & $1024(80.5)$ \\
Yes & $39.9(8.1)$ \\
No & $715(61.9)$ \\
Hours worked per week, mean (SD) & \\
Practice size & $169(14.6)$ \\
$\leq 5$ clinicians & \\
6-10 & $575(51.4)$ \\
$\geq 11$ & $346(30.9)$ \\
Medically underserved area & $197(17.6)$ \\
No & \\
Yes & $691(60.2)$ \\
Do not know & $457(39.8)$ \\
Practice's specialty mix &
\end{tabular}

*Advanced Practice Clinicians includes nurse practitioners and physician assistants

${ }^{\dagger}$ Clinical support staff includes registered nurse, licensed practical nurse and medical assistants

${ }^{H A d m i n i s t r a t i v e ~ s t a f f ~ i n c l u d e s ~ o f f i c e ~ m a n a g e r s, ~ r e c e p t i o n i s t s, ~ b i l l i n g ~}$ staff and other

${ }^{\xi}$ Note that frequencies do not add to 1279 due to missing data consideration in addressing burnout in primary care. Individual response to changes in the work environment and workplace demands, such as role modifications and task complexity, may influence job satisfaction, ambition and commitment to the organization. In this study, we used the Change Diagnostic Index@ (CDI) instrument to capture individual behavioural response to change in primary care practices. ${ }^{27,28}$ The CDI was designed to study employee reaction to organizational or technological change such as an organizational merger or the adoption of EHRs. The CDI focuses on the nature and intensity of attitudes, perceptions and behaviours that arise due to factors associated with a change initiative. Individual reactions can manifest into adverse organization-wide behaviours that include decreased efficiency, morale or motivation, or increased conflict, absenteeism and turnover.

The CDI instrument is based on a 5-point Likert-type scale (levels ranged from strongly agree to strongly disagree) and consists of 25 questions in seven domains: anxiety, frustration, delayed development, rejection of the environment, refusal to participate, withdrawal and global. Individuals in this study were instructed to respond to the CDI questions based on their involvement in the HVH initiative, which involved changes in work processes and functions.

CDI domains are based on a continuum; for example, individuals might experience more or less anxiety, more or less frustration, or feel more or less threatened in their current job environment. One example of a CDI question is "It is disruptive to me that so many changes are occurring at my place of employment." We reverse-coded the items so that both the CDI and PAR were in the same direction. The average summed responses of all 25 reverse-coded items were used in data analyses.

\section{Data Analysis}

We used descriptive statistics to analyse sample characteristics. The $t$ test was used to assess significant differences in the 
average values of PAR and CDI between those who expressed burnout versus those who did not by type of healthcare professional and for the entire sample. Multivariable logistic regression models were built to estimate the effects (computed odds ratios and $95 \%$ confidence intervals) of PAR and CDI on the likelihood of burnout for the entire sample and by different types of healthcare professionals, adjusting for hours worked per week and practice characteristics. We entered an interaction term between the centered average values of PAR and CDI in the statistical models to examine whether PAR moderates the relationship between CDI and the likelihood of a healthcare professional's report of burnout. For significant interaction terms, conditional effects were computed at three values of CDI: $1 \mathrm{SD}$ below the mean, at the mean, and $1 \mathrm{SD}$ above the mean. The logistic regression models were adjusted for clustered standard errors at the practice level. Stata software version 14 was used in the analyses.

\section{RESULTS}

Approximately $20 \%$ of healthcare professionals in our study reported burnout; see Table 1 . The average number of hours worked per week was $40(\mathrm{SD}=8.1)$ hours. Most healthcare professionals $(60.2 \%)$ worked at practices classified as single specialty, and one-third were located in a medically underserved area. The results in Table 2 show the distribution of burnout among providers, clinical support staff and administrative staff. Reports of burnout were higher among providers relative to other healthcare professionals. Specifically, 25.5\% of the providers reported burnout, followed by $18.9 \%$ of the clinical support staff, and $17.5 \%$ of the administrative staff.

Overall, there were significant differences $(p$ value $<0.01)$ in the average scores for both CDI and PAR between reports of burnout and no burnout by type of healthcare professional; see Table 3. Specifically, healthcare professionals who reported no burnout had significantly higher scores of CDI (positive behavioural response toward change) and worked in practices with higher scores on PAR (organizational capacity for change).

The logistic regression model for the entire sample revealed significant effects of PAR and CDI on burnout; see Table 4. For every one score increase in average PAR, the adjusted odds of burnout decreased by a factor of $0.49(0.33,0.73)$. In other words, as the change capacity of a practice (PAR) increases, burnout in healthcare professionals decreased significantly by $51 \%$. Similarly, as the healthcare professionals showed improved response toward change as measured by the CDI, burnout decreased by $84 \%$ (OR 0.16 ; 95\% CI, 0.11 , 0.23 ). Our analysis also revealed a significant moderation effect of PAR on the relationship between CDI and burnout (OR 1.67; 95\% CI, 1.02, 2.74). In order to examine further this interaction, conditional effects were computed for $1 \mathrm{SD}$ below the mean, at the mean, and $1 \mathrm{SD}$ above the mean for PAR. Our findings revealed that at increasing practice adaptability levels, i.e. higher values of PAR, higher levels of CDI significantly decreased burnout. Increased hours of work per week, larger practice size ( $\geq 11$ clinicians) and single specialty were associated with higher odds of burnout reports in healthcare professionals.

When separate logistic regression models were built based on the type of healthcare professional, we did not find any significant effects of PAR on burnout among providers or administrative staff. However, higher levels of PAR significantly lowered the odds of burnout reports by $0.21(0.10,0.46)$ in clinical support staff. Increasing average levels of CDI were associated with a statistically significant, but small, decrease in burnout for providers (OR: $0.05 ; 95 \% \mathrm{CI}, 0.01,0.19)$, clinical support staff (OR: $0.22 ; 95 \%$ CI, 0.12, 0.39) and administrative staff (OR: $0.12 ; 95 \% \mathrm{CI}, 0.07,0.24)$. The moderation effect of PAR on the relationship of CDI and burnout was significant for the administrative staff (OR: 2.92; 95\% CI, $1.37,6.24)$. Furthermore, increasing the number of hours worked per week significantly increased the likelihood of burnout across all healthcare professional groups. Single specialty practice was significantly associated with $3.70(1.62$, 8.45) higher odds of burnout in clinical support staff.

\section{DISCUSSION}

This study found that higher levels of adaptive reserve and positive behavioural response to change among healthcare professionals are associated with lower burnout. Results also demonstrate that adaptive reserve has a moderating effect on the relationship between behavioural response to change and burnout when healthcare professionals are analysed together. This indicates that a greater personal ability to respond to change is associated with lower burnout when the work environment is characterized by strong communication systems, teamwork, leadership support, and other traits of adaptive reserve. Analysis by healthcare professional type, however, revealed that this moderating effect was not present among providers and clinical support staff. Another finding from our study is that increasing the number of hours worked per week was significantly related to burnout across healthcare professional groups.

Our findings, combined with the results from previous studies, indicate that when primary care practices are equipped to improve their capacity to adopt and sustain change, and this can also improve healthcare professionals' response to change and lead to reduced reports of burnout. Health system and practice leaders who wish to increase adaptive reserve should focus on building positive work environments through interprofessional teamwork, employee engagement and enhanced communication.

Team-based care, a component of adaptive reserve, has been found to improve clinical outcomes and reduce levels of burnout. ${ }^{29}$ A leading example of team-based care is the Primary Care Redesign model from the University of 
Table 2 Burnout among Physicians, Advanced Practice Clinicians, Clinical Support Staff and Administrative Staff

\begin{tabular}{|c|c|c|c|c|}
\hline & \multicolumn{3}{|c|}{ Healthcare profession } & \multirow{2}{*}{$\begin{array}{l}\text { Tota } \\
(\%)\end{array}$} \\
\hline & $\begin{array}{l}\text { Providers }{ }^{\dagger}, n= \\
238(\%)\end{array}$ & $\begin{array}{l}\text { Clinical support } \\
\text { staff }^{\dagger}, n=451(\%)\end{array}$ & $\begin{array}{l}\text { Administrative staff }{ }^{\S}, n \\
=578(\%)\end{array}$ & \\
\hline \multicolumn{5}{|l|}{ Burnout* } \\
\hline Yes & 25.5 & 18.9 & 17.5 & 19.5 \\
\hline No & 74.5 & 81.1 & 82.5 & 80.5 \\
\hline \multicolumn{5}{|l|}{ Specific components of burnout** } \\
\hline 1. I enjoy my work. I have no symptoms of burnout. & 25.5 & 35.1 & 34.1 & 32.9 \\
\hline $\begin{array}{l}\text { 2. Occasionally I am under stress, and I don't always have as } \\
\text { much energy as I once did, but I don't feel burned out. }\end{array}$ & 48.9 & 46.0 & 48.4 & 47.7 \\
\hline $\begin{array}{l}\text { 3. I am definitely burning out and have one or more } \\
\text { symptoms of burnout, such as physical and emotional } \\
\text { exhaustion. }\end{array}$ & 17.4 & 12.7 & 13.7 & 14.0 \\
\hline $\begin{array}{l}\text { 4. The symptoms of burnout that I'm experiencing won't go } \\
\text { away. I think about frustrations at work a lot. }\end{array}$ & 4.3 & 4.0 & 2.2 & 3.2 \\
\hline $\begin{array}{l}\text { away. I think about frustrations at work a lot. } \\
5 \text {. I feel completely burned out and often wonder if I can go } \\
\text { on practicing. I am at the point where I may need some } \\
\text { changes. }\end{array}$ & 3.8 & 2.2 & 1.6 & 2.2 \\
\hline
\end{tabular}

*Burnout was determined by a response of 3, 4 or 5, on the question above

${ }^{\dagger}$ Providers includes physicians, nurse practitioners and physician assistants

${ }^{H C}$ Clinical support staff includes registered nurses, licensed practical nurses and medical assistants

$\S_{\text {Administrative staff includes office managers, receptionists, billing staff and other }}$

Colorado, which includes expanded roles for medical assistants (MAs), team-based documentation and delegation of tasks such as medication reconciliation and refills. This model resulted in improvements in quality, access, clinician experience and reductions in burnout. ${ }^{30}$ Another team-based care model that focused on expanding roles for nurses and other staff resulted in improved clinical outcomes, increased revenues and higher physician and staff satisfaction. ${ }^{31}$

Constructive communication and support among team members may optimize how individuals respond to stress and perform work-related tasks. One previous study in primary care found that redesigning workflows, improving communication among physicians and staff, and considering physician input significantly reduced burnout and dissatisfaction, and improved retention. ${ }^{20}$ Practice leaders should develop a communication strategy during major change initiatives and administer frequent pulse surveys to obtain employee feedback. In addition to administering surveys such as those used in our study, practice leaders should consider holding meetings with employees to communicate information about major practice changes, gain an overall impression of employee morale and their concerns and obtain input on the implementation of the change.

The finding of an association between the number of hours worked per week and burnout across healthcare professional types is an important consideration for efforts to improve workplace wellness. Previous research found that team staffing levels, patient panel size and the number of hours worked per week were associated with burnout among physicians. ${ }^{32-34}$ This highlights the need for practices to accurately

Table 3 Differences in Average Change Diagnostic Index@ (CDI) and Practice Adaptive Reserve (PAR) by Burnout Status in Healthcare Professionals

\begin{tabular}{|c|c|c|c|c|}
\hline & \multirow{2}{*}{$\begin{array}{l}\text { Total } \\
\text { Mean (SD) }\end{array}$} & \multicolumn{2}{|l|}{ Burnout } & \multirow[t]{2}{*}{$p$ value } \\
\hline & & Yes, $n=249$, mean $(\mathrm{SD})$ & No, $n=1024$, mean $(\mathrm{SD})$ & \\
\hline \multicolumn{5}{|l|}{ Sample } \\
\hline $\mathrm{CDI}^{\dagger}$ & $4.00(0.61)$ & $3.45(0.58)$ & $4.14(0.54)$ & $<0.001$ \\
\hline Practice adaptive reserve & $3.76(0.66)$ & $3.23(0.71)$ & $3.89(0.58)$ & $<0.001$ \\
\hline \multicolumn{5}{|l|}{ Providers ${ }^{\dagger \dagger}$} \\
\hline $\mathrm{CDI}^{\dagger}$ & $3.98(0.60)$ & $3.46(0.56)$ & $4.16(0.50)$ & $<0.001$ \\
\hline Practice adaptive reserve & $3.78(0.56)$ & $3.43(0.55)$ & $3.92(0.49)$ & $<0.001$ \\
\hline \multicolumn{5}{|l|}{ Clinical support staff ${ }^{\S}$} \\
\hline $\mathrm{CDI}^{\dagger}$ & $3.98(0.63)$ & $3.44(0.58)$ & $4.11(0.57)$ & $<0.001$ \\
\hline Practice adaptive reserve & $3.73(0.69)$ & $3.06(0.72)$ & $3.88(0.58)$ & $<0.001$ \\
\hline \multicolumn{5}{|l|}{ Administrative staff ${ }^{\mid}$} \\
\hline $\mathrm{CDI}^{\dagger}$ & $4.03(0.60)$ & $3.45(0.58)$ & $4.15(0.53)$ & $<0.001$ \\
\hline Practice adaptive reserve & $3.78(0.65)$ & $3.29(0.69)$ & $3.87(0.59)$ & $<0.001$ \\
\hline
\end{tabular}

*The $p$ value is based on t test analyses testing for significant differences in CDI and PAR mean scores between burnout and non-burnout for the entire sample and by type of healthcare professionals

${ }^{\dagger} C D I$ is the Change Diagnostic Index. (C)

Providers includes physicians, nurse practitioners and physician assistants

$\$$ Clinical support staff includes registered nurses, licensed practical nurses and medical assistants.

I Administrative staff includes office managers, receptionists, billing staff and other 
Table 4 Adjusted Logistic Regression (Odds Ratios and 95\% Confidence Intervals) for Burnout in Healthcare Professionals based on the Change Diagnostic Index@ (CDI) and Practice Adaptive Reserve (PAR)

\begin{tabular}{|c|c|c|c|c|}
\hline & $\frac{\text { Entire sample burnout }}{\text { OR }(95 \% \mathrm{CI})}$ & $\frac{\text { Provider* burnout }}{\text { OR }(95 \% \mathrm{CI})}$ & $\frac{\text { Clinical support staff }{ }^{\dagger} \text { burnout }}{\text { OR }(95 \% \text { CI })}$ & $\begin{array}{l}\text { Administrative Staff }{ }^{\dagger \dagger} \text { burnou } \\
\text { OR (95\% CI) }\end{array}$ \\
\hline Practice adaptive reserve & $0.49(0.33,0.73)$ & $0.53(0.18,1.57)$ & $0.21(0.10,0.46)$ & $1.14(0.66,1.99)$ \\
\hline Adaptive reserve $\mathrm{x} \mathrm{CDI}^{\dagger}$ & $1.67(1.02,2.74)$ & $2.57(0.33,19.8)$ & $1.03(0.54,1.96)$ & $2.92(1.37,6.24)$ \\
\hline Hours worked per week & $1.07(1.05,1.10)$ & $1.09(1.04,1.15)$ & $1.06(1.01,1.11)$ & $1.06(1.01,1.12)$ \\
\hline \multicolumn{5}{|l|}{ Practice size } \\
\hline$\leq 5$ clinicians & Reference & & Reference & Reference \\
\hline \multicolumn{5}{|c|}{ Medically underserved area } \\
\hline No & $0.93(0.53,1.62)$ & $1.12(0.29,4.26)$ & $0.48(0.21,1.14)$ & $1.86(0.75,4.59)$ \\
\hline Yes & Reference & Reference & Reference & Reference \\
\hline Do not know & $1.14(0.57,2.28)$ & $1.14(0.27,4.73)$ & $0.66(0.21,2.03)$ & $1.91(0.76,4.85)$ \\
\hline \multicolumn{5}{|l|}{ Practice's specialty mix } \\
\hline Single specialty & $1.84(1.04,3.36)$ & $1.35(0.44,4.07)$ & $3.70(1.62,8.45)$ & $1.18(0.56,2.49)$ \\
\hline Multispecialty & Reference & Reference & Reference & Reference \\
\hline
\end{tabular}

*Providers include physicians, nurse practitioners and physician assistants

${ }^{\dagger}$ Clinical support staff includes registered nurses, licensed practical nurses and medical assistants

${ }^{1+}$ Administrative staff includes office managers, receptionists, billing staff and other

${ }^{\xi_{C}} \mathrm{CDI}$ is the Change Diagnostic Index. (C)

Findings highlighted in italics were statistically significant at $p$ value $\leq 0.05$

assess the complexity of work and the total workload expected of healthcare professionals. Monitoring is particularly important during major change initiatives that affect healthcare professionals' job demands, work complexity and resources to complete job assignments, such as useful and usable technologies. ${ }^{1}$

Our finding that adaptive reserve (practice capacity for change) was only a moderator on the relationship between behavioural response to change and burnout among administrative staff is important. This could reflect a decrease in autonomy ${ }^{35}$ among providers and clinical staff, in which they perceive to have limited capacity to shape changes they face in the workplace, regardless of the organizational systems and structure. This finding may also indicate that a portion of burnout for these healthcare professionals is related to moral distress, which is a misalignment between personal or professional values and those of the practice or health system. ${ }^{36}$ Our study supports the need to understand how changes in the work environment influence healthcare professionals and their behavioural response to change. Additional research is needed to identify personal, organizational and societal interventions to reduce burnout among providers and other healthcare professionals. ${ }^{37}$

This study has several limitations that should be considered. The use of cross-sectional data in the current study does not allow for causal inferences. Study data were collected from physicians, advanced practice clinicians and staff at primary care practices in Virginia and may not be generalized to other regions. In addition, practices who volunteered for the $\mathrm{HVH}$ study may exhibit characteristics different from other practices, which may result in a selection bias. Data were obtained from a self-report survey, which may produce response bias based on social desirability, extreme or mid-point response style, or other response behaviours that may affect measurement quality. The significant moderation effect of PAR and CDI on burnout found in this study for the entire sample and separately for administrative staff may not hold true for different samples and/or at different times of assessment for these healthcare professionals. This study should be repeated in a larger and more geographically diverse sample of health care professionals. Future studies should also evaluate the association between each PAR domain and burnout among different healthcare professionals.

As transformation in primary care practices continues, it is critical to understand the influence of these initiatives on healthcare professionals' well-being. Over the past several decades, there have been considerable changes to working conditions, organizational climate, job responsibilities and work demands in primary care. These conditions contribute to burnout, along with numerous other context-dependent factors, which underscores the need for multi-pronged strategies at both the system and organizational levels. Our study provides empirical evidence that supports the need for policymakers and organizational leaders to consider individual and organizational factors in developing strategies to reduce burnout. Initiatives that include strategies to promote interprofessional teamwork and communication among team members may reduce stress and foster healthier workplaces. Now, more than ever, there needs to be major improvements in work environments to support healthcare professionals in primary care settings.

Corresponding Author: Debora Goetz Goldberg, PhD, MHA, MBA; Department of Health Administration and Policy, George Mason University, Fairfax, VA, USA (e-mail: dgoldbe4@gmu.edu).

Funding This study was funded by the Agency for Healthcare Research and Quality (AHRQ) under grant number R18HSO23913. 


\section{Compliance with Ethical Standards:}

Conflict of Interest: D. Goldberg, T. Soylu, P. Kitsantas, and L. Nichols report no conflict of interest. $V$. Grady reports proprietary interest in the Change Diagnostic Index(C) instrument.

\section{REFERENCES}

1. National Academy of Medicine. Taking action against clinician burnout, A systems approach to professional well-being. Washington, DC; 2019.

2. Goldberg DG, Kuzel AJ, Feng LB, Love LE. EHRs in primary care practices: benefits, challenges, and successful strategies. Am J Manag Care 2012;18(2): e48-54.

3. Meyers DS, Clancy CM. Primary care: Too important to fail. Ann Intern Med 2009; 150:272-273.

4. Nachimson Advisors, LLC. The cost of implementing ICD-10 for physician practices - updating the 2008 Nachimson Advisors study. A report to the American Medical Association. 2014. Available from: https://docs.house. gov/meetings/IF/IF 14/20150211/102940/HHRG-114-IF 14-WstateTerryW-20150211-SD001.pdf. Accessed 15 Dec 2020.

5. Sinsky, C, Willard-Grace R, Schutzbank AM, Sinsky T, Margolius D, Bodenheimer T. In search of joy in practice: A report of 23 highfunctioning primary care practices. Ann Fam Med 2013; 11(3): 272-278.

6. Grumbach $\mathbf{K}$, Knox M, Huang $\mathbf{B}$, Hammer $\mathbf{H}$, Kivlahan $\mathbf{C}$, WillardGrace R. A longitudinal study of trends in burnout during primary care transformation. Ann Fam Med 2019;17(Suppl 1):S9-S16.

7. Casalicchio G, Lesaffre E, Küchenhoff $\mathbf{H}$, Bruyneel L. Nonlinear analysis to detect if excellent nursing work environments have highest well-being. J Nurs Scholarsh 2017;49(5):537-547.

8. Hanrahan NP, Aiken LH, McClaine L, Hanlon AL. Relationship between psychiatric nurse work environments and nurse burnout in acute care general hospitals. Issues Ment Health Nurs 2010;31(3): 198-207.

9. Hayes B, Douglas C, Bonner A. Work environment, job satisfaction, stress and burnout among haemodialysis nurses. J Nurs Manag 2015;23:588- 598.

10. Maslach C, Jackson SE, Leiter MP. MBI: The Maslach Burnout Inventory: Manual. Consulting Psychologists Press, Palo Alto, CA; 1996.

11. Dewa CS, Loong D, Bonato S, Trojanowski L. The relationship between physician burnout and quality of healthcare in terms of safety and acceptability: a systematic review. BMJ Open 2017;7(6):e015141.

12. Linzer M, Manwell LB, Williams ES, et al. Working conditions in primary care: physician reactions and care quality. Ann Intern Med 2009; 151(1):28-36, W6-9.

13. Shanafelt TD, Bradley KA, Wipf JE, Back AL. Burnout and selfreported patient care in an internal medicine residency program. Ann Intern Med 2002;136(5):358-367.

14. Shanafelt TD, Balch CM, Bechamps G, et al. Burnout and medical errors among American surgeons. Ann Surg 2010;251(6):995-1000.

15. Goldberg DG, Soylu TG, Grady V, Kitsantas P, Nichols L. Indicators of workplace burnout among physicians, advanced practice clinicians and staff in small to medium-sized primary care practices. J Am Board Fam Med 2020;33(3):378-385.

16. Shanafelt TD, West CP, Sinsky C, et al. Changes in burnout and satisfaction with work-life integration in physicians and the general US working population between 2011 and 2017. Mayo Clin Proc 2019;94(9):1681-1694.

17. Jha AK, Miff AR, Chaoui AA, Defossez S, Bombaugh MC, Miller YA. A crisis in health care: A call to action on physician burnout. Massachusetts Medical Society; 2018. Available from: http://www.massmed.org/Newsand-Publications /MMS-News-Releases /Physician-Burnout-Report2018/. Accessed 15 Dec 2020.
18. US Department of Health and Human Services, Agency for Healthcare Research and Quality (AHRQ). EvidenceNow: advancing heart health in primary care. Available from: https://www.ahrq.gov/evidencenow/index. html. Jun 10, 2017. Accessed 15 Dec 2020.

19. Cuellar A, Krist AH, Nichols LM, Kuzel A. Effect of practice ownership on work environment, learning culture, psychological safety, and burnout. Ann Fam Med 2018;16(Suppl 1):S44-S51.

20. Linzer M, Poplau S, Grossman E, et al. A cluster randomized trial of interventions to improve work conditions and clinician burnout in primary care: results from the Healthy Work Place (HWP) study. J Gen Intern Med 2015;30(8):1105-1011.

21. Dolan ED, Mohr D, Lempa M, et al. Using a single item to measure burnout in primary care staff: a psychometric evaluation. J Gen Intern Med 2015;30(5):582-587.

22. Rohland BM, Kruse GR, Rohrer JE. Validation of a single-item measure of burnout against the Maslach Burnout Inventory among physicians. Stress Health 2004; 20(2):75-79.

23. Miller WL, Crabtree BF, Nutting PA, Stange KC, Jaén CR. Primary care practice development: a relationship-centered approach. Ann Fam Med 2010;8(Suppl 1):S68-S79, S92.

24. Blechter B; Jiang N; Cleland C; Berry C; Ogedegbe O; Shelley D. Correlates of burnout in small independent primary care practices in an urban setting. J Am Board Fam Med 2018;31(4):529-536.

25. Huynh C, Bowles D, Shan MY, et al. Change implementation: the association of adaptive reserve and burnout among inpatient medicine physicians and nurses. J Interprof Care 2018;32(5):549-555.

26. Willard-Grace R, Hessler D, Rogers E, Dubé K, Bodenheimer T, Grumbach $\mathbf{K}$. Team structure and culture are associated with lower burnout in primary care. J Am Board Fam Med 2014; 27(2):229-238.

27. Grady V, Grady J. The relationship of Bowlby's attachment theory to the persistent failure of organizational change initiatives. J Chang Manag 2012;13(2):206-222.

28. Grady V, Grady J. The pivot point: Success in organizational change. New York, NY: Morgan-James Publishing; 2013.

29. DeChant PF, Acs A, Rhee KB, et al. Effect of Organization-Directed Workplace Interventions on Physician Burnout: A Systematic Review. Mayo Clin Proc Innov Qual Outcomes 2019;3(4):384-408.

30. Smith PC, Lyon C, English AF, Conry C. Practice transformation under the University of Colorado's Primary Care Redesign model. Ann Fam Med 2019; 17(Suppl 1):S24-S32.

31. Anderson P, Halley MD. A new approach to making your doctor-nurse team more productive. Fam Pract Manag 2008;15:35-40.

32. Rassolian M, Peterson LE, Fang B, et al. Workplace factors associated with burnout of family physicians. JAMA Intern Med 2017; 177(7):10361038.

33. Hu NC, Chen JD, Cheng TJ. The associations between long working hours, physical inactivity, and burnout. J Occup Environ Med 2016; 58(5):514-518.

34. Helfrich, CD, Simonetti, JA, Clinton, WL, et al. The association of team-specific workload and staffing with odds of burnout among VA primary care team members. J Gen Intern Med 2017;32:760-766.

35. Friedberg MW, Chen PG, Van Busum KR, et al. Factors affecting physician professional satisfaction and their implications for patient care, health systems, and health policy. Rand Health $\mathrm{Q}$ 2014;3(4): 1.

36. Johnson-Coyle L, Opgenorth D, Bellows M, Dhaliwal J, RichardsonCarr S, Bagshaw SM. Moral distress and burnout among cardiovascular surgery intensive care unit healthcare professionals: A prospective crosssectional survey. Can J Crit Care Nurs 2016;27(4):27-36.

37. Peikes DN, Swankoski K, Hoag SD, et al. The effects of a primary care transformation initiative on primary care physician burnout and workplace experience. J Gen Intern Med 2019; 34(1): 49-57.

Publisher's Note: Springer Nature remains neutral with regard to jurisdictional claims in published maps and institutional affiliations. 